\title{
PLANNING SEAMLESS WI-FI NETWORKS WITH CONSIDERATION FOR THE IMPACT OF INTRASYSTEM INTERFERENCE
}

\author{
Ainur A. Ziganshin, \\ Dept. of Radioelectronic and Telecommunication Systems \\ Kazan National Research Technical University named after A. N. Tupolev-KAI, \\ City of Kazan, Russian Federation \\ ZiganshinAA@stud.kai.ru \\ Ekaterina A. Petrova, \\ Dept. of Radioelectronic and Telecommunication Systems \\ Kazan National Research Technical University named after A. N. Tupolev-KAI, \\ City of Kazan, Russian Federation \\ EAPetrova@kai.ru
}

DOI: $10.36724 / 2664-066 X-2020-6-\mid-23-28$

\begin{abstract}
Most wireless networks operate under the influence of intrasystem interference, which reduces the data rate. The purpose of this work is to create an algorithm for optimizing a seamless network with consideration for intrasystem interference. For this purpose, a general optimization technique was developed, including a vector of optimized parameters and equations necessary for calculating the target function. The developed method was demonstrated on the example of the RTS Department network. The result was the reduction of the network load and increase network throughput.
\end{abstract}

KEYWORDS: seamless Wi-Fi, optimization, signal to interference plus noise ratio, signal to noise ratio, intrasystem interference.

\section{INTRODUCTION}

An important step in designing seamless Wi-Fi networks is to optimize network parameters. Since the zones of different access points overlap in seamless Wi-Fi networks, the impact of intrasystem interference must be taken into account when optimizing network parameters [1].

In Wi-Fi network, we can distinguish two types of radio interference: co-channel and adjacent channels. Interference on adjacent channels can take on large values, especially for seamless Wi-Fi networks where access points are located close enough. Most programs similar to "Ekahau site suite" [2] only take into account co-channel interference, which gives not quite accurate results. Therefore, considering both these factors into account allows designing network more effectively [3]. 


\section{Problem Statement}

Consider an arbitrary seamless Wi-Fi network, which according to the preliminary plan serves the territory $S^{\text {net }}$ specified by its floor plan and consisting of $M$ mobile subscriber devices and $L$ access points. Requirements of subscribers are known. There is also information about where the base stations are initially located and what their parameters are.

Floor plans can be set as two-dimensional (2D) drawings or as three-dimensional (3D) objects. The 2D plan sets the location of subscribers and access points, and sets the size of the walls. In this case, data processing is fast, however offices often have partitions and walls that do not reach the ceiling. For such offices, it is difficult to apply 2D plan, so its need to use the plan with $3 \mathrm{D}$ objects.

The 3D plan also sets the location of subscribers and access points, and sets the walls as a set of planes. Algorithms with a large amount of calculations are used to determine the crossing with walls therefore the processing time will be longer.

The optimization task is to determine the parameters of access points that meet the requirements for all network subscribers.

For each access point, the parameters that can be changed are the coordinates of the installation $\left(x_{l}, y_{l}\right)$, the height of the suspension $h_{l}$, then azimuth $\alpha_{l}$ and angle of elevation direction $\beta_{l}$, the maximum transmitted power $P_{l}^{\max }$, the antenna gain $G_{l}$, and the radiation pattern $F(\theta, \varphi)$. By manipulating these parameters, we need to achieve optimal network parameters, taking into account the intrasystem interference.

Hence, the vector of optimized parameters includes number of access points $L$, territory $S^{\text {net }}$ and individual vectors of parameters for each access point:

$\vec{V}=\left(L, S^{n e t}, \vec{V}_{1}, \vec{V}_{2}, \ldots \vec{V}_{L}\right)$,

where $\vec{V}_{l}=\left(x_{l}, y_{l}, h_{l}, P_{l}^{\max }, G_{l}, F_{l}(\theta, \varphi), \alpha_{l}, \beta_{l}\right)$.

\section{MethOD}

High data rate is not a guarantee that the users will get that they need. We also need take into account the number of customers connecting to access point. Therefore, we consider network loads $A^{\text {net }}$ [4].

The network load is determined by maximal value of loads on the access point $A_{l}, l=\overline{1, L}[4]$ :

$$
A^{\text {net }}=\max _{l=1, L}\left\{A_{l}\right\}
$$

If $A^{\text {net }}<1$, then there is a time margin and it is possible to transmit more data. If $A^{\text {net }}>1$, then all data cannot be transmitted, in this case the information rate will decrease.

The load on the access point $A_{l}$ is defined as sum of loads $A_{l m}$ created by subscriber devices connected to this point [5]:

$$
A_{l}=\sum_{m=1}^{M} A_{l m}
$$

After calculating the load on each access point, we will select maximum from them.

The subscriber load is the fraction of time necessary to deliver data to the subscriber. The subscriber will connect to the access point from which it has the maximum data rate. In this case, subscriber load can be defined as ratio of real data transfer rate to the specified one [6].

$A_{l m}=\left\{\begin{array}{l}\frac{V_{m}^{\text {sprc }}}{V_{l m}^{\text {real }},} \text { if } l=\arg \max _{l^{\prime}=1, L}\left\{V_{l m}^{\text {real }}\right\} \\ 0, \quad \text { if } l \neq \arg \max _{l^{\prime}=1, L}\left\{V_{l m}^{\text {real }}\right\}\end{array}\right.$,

where $V_{m}^{\text {spec }}$ - specified data rate to $m$-th subscriber device, $V_{l m}^{\text {real }}$ - real data rate that the $m$-th subscriber device can get from $l$-th access point. The specified rate is determined based on the quality of services provided to the subscriber device.

This selection is made by the seamless network controller that manages the wireless network. The choice is based on data rate, not signal strength, since speed is not always monotonously dependent on signal strength, for example due to strong intrasystem interference.

Data rate of OFDM signal depends on number of subcarriers, symbol duration, signal to interference plus noise ratio (SINR) for each subcarrier and connected access point [7]:

$$
V_{l m}^{\text {real }}\left(R^{S}\right) \approx 2 \cdot V^{\text {tech }} \cdot \sum_{i=1}^{I^{u}} \log _{2}\left(\frac{\sqrt{\rho_{i l m}^{R^{S}}}}{2 \cdot Q^{-1}\left(P_{\max }^{E r} / 2\right)}+1\right),
$$

where $V^{\text {tech }}$ - technical data rate, $Q^{-1}(\bullet)$ - inverse Q-function, $P_{\max }^{E r}-$ maximum possible error probability, $I^{u}$ - number of information subcarriers, $\rho_{i l m}^{R^{S}}-$ SINR, $R^{S}$ - signal receiving algorithm.

$$
Q(\rho)=\frac{1}{\sqrt{2 \cdot \pi}} \int_{\rho}^{\infty} e^{-\frac{x^{2}}{2}} d x
$$


$Q(\rho)$ is the probability that a standard normal random variable takes a value larger than $\rho$.

Technical data rate $V^{\text {tech }}$ is the number of OFDM symbols transmitted per second. It depends on duration of the OFDM symbol.

Number of information subcarriers $I^{u}$ depend on the generation of Wi-Fi used and the width of the frequency channel.

Maximum possible error probability $P_{\max }^{E r}$ determines the error probability above which the transfer can be considered invalid and incorrect.

\section{HOW THE SINR AND SNR IS DEFINED}

Each subcarrier selects its own data rate according to its SINR. With a strong signal level, it is possible to modulate at high rate with a large number of elements in the signal constellation. For a low strength signal, can use more noise-tolerant encoding with lower rate. We can define SINR as [8]:

$$
\rho_{i l m}^{R^{S}}=\frac{\rho_{l m}}{1+K_{i}^{N R^{S}}+\sum_{l^{\prime}=1}^{L} \rho_{l^{\prime} m} \cdot K_{i l l^{\prime}}^{\Sigma P R^{S}}},
$$

where $\rho_{l m}-$ SNR of data transmitting $l$-th access point for $m$-th subscriber device, $\rho_{l^{\prime} m}-$ SNR of the interference $l^{\prime}$-th access point on for $m$-th subscriber device, $K_{i}^{N R^{S}}$ - noise coefficient for $i$-th subcarrier, $K_{i l l^{\prime}}^{\Sigma P R^{S}}$ - the total coefficient of intrasystem interference on the $i$-th subcarrier.

Total coefficients of intrasystem interference show how signal is also consisting from many OFDM subcarriers channel affects to our signal:

$$
K_{i l l^{\prime}}^{\Sigma P R^{S}}=\sum_{i^{\prime}=1}^{I^{A}} K_{i l i^{\prime} l^{\prime}}^{\Sigma P R^{S}}
$$

where $K_{i l l^{\prime} l^{\prime}}^{\sum P R^{S}}$ - interference caused by a subcarrier $i^{\prime}$ from station $l^{\prime}, I^{A}$ - number of active OFDM subcarriers.

For the applied FFT-based receiving algorithm, according to [9], the noise coefficient is zero.

The coefficients of total intrasystem interference are calculated for the 802.11n standard with "OFDM Receiver" program [10].

SNR of transmitting $l$-th access point for $m$-th subscriber device is calculated as: $\rho_{l m n}=\frac{P_{l m}}{P_{n}}$,

where $P_{l m}$ - power of $l$-th access point at input of $m$-th subscriber device, $P_{n}$ - noise power.

We can take the heat noise level of the receiver as the noise power. If you have the right equipment, you can determine the level of white noise and consider it in the calculations. This method allows to take these options into account.

We need to determine the signal strength $P_{l m}$. The signal from the transmitter fades when propagated in the in the medium. To calculate the signal strength, we must also take into account the direction of the antenna.

$P_{l m}=P_{l}-L_{l m}+G_{l}+F_{l}\left(\theta_{l m}, \varphi_{l m}\right)(d B m)$,

where $P_{l}$ - transmitter power, $L_{l m}$ - propagation loss between $l$-th access point and $m$-th subscriber device, $G_{l}$ - gain, $F_{l}\left(\theta_{l m}, \varphi_{l m}\right)$ - radiation pattern of $l$-th access point in the direction of $m$-th subscriber device.

The calculation of losses is made in accordance with the recommendation ITU-R P.1238-8 [11]. We take into account the distance, the number of crossed walls, and the frequency of the signal.

$L_{l m}(d B)=20 \cdot \lg \left(f_{l}\right)+N \cdot \lg \left(d_{l m}\right)+L_{f}\left(n_{l m}\right)-28$,

where $f_{l}$ - frequency of signal, $N-$ power loss coefficient at a distance, $d_{l m}$ - distance between the $l$-th access point and the $m$-th subscriber device, $L_{f}(\bullet)-$ loss factor due to signal passing through the floor or wall, $n_{l m}$ - number of crossing walls and floors between the $l$ th access point and the $m$-th subscriber device.

Gain $G_{l}$ and radiation pattern $F(\theta, \varphi)$ depend on the type of antenna used in the access point as well as the direction of its axis which is determined by angles of azimuth $\alpha_{l}$ and elevation $\beta_{l}$

To calculate losses and determine $P_{l m}$, it's necessary to know the location of subscribers in the served territory.

\section{PLACEMENT IN THE WORST POINTS}

Our users are mobile, so we will assume that they are located in places where data rate is minimal since at these points they will create the maximum load. If they get acceptable conditions in these places, their data rate will be higher in other places. 
After calculating the speeds at all points, we can choose from them $M$ points and place subscribers in them and find the load.

Thus, for each value of the vector of optimized parameters $\vec{V}$, we can determine the loads on access points and load on the network $A^{\text {net }}$.

\section{CRITERION}

To ensure that the requirements are met for all network subscribers, the following condition is required $A^{\text {net }}<1$.

Based on this, taking into account the margin in the load, the optimization criterion can be written as a minimum load on the network $A^{\text {net }}$ :

$$
\vec{V}^{\text {opt }}=\arg \min _{\langle\vec{V}\rangle}\left\{A^{\text {net }}(\vec{V})\right\} .
$$

\section{FORMULATION OF THE OPTIMIZATION PROBLEM}

By changing the parameter values of the optimization vector (1), its need to find such values of this vector that the load on the network will be as small as possible. There are many optimization methods and algorithms available. Some of them are looking for a global minimum, while others are looking for a local minimum. Among them, we need to choose the appropriate one to solve the task.

\section{CHOOSING AN OPTIMIZATION ALGORITHM}

Multi-dimensional optimization methods are used for optimization of several parameters. The most well-known methods are those related to finding the gradient. In our case, finding the derivative is impossible due to the discreteness of the coordinate step and the presence of obstacles. Therefore, we need numerical optimization methods [12]. It is also possible to use non-linear programming. Using this method, the values of target functions are calculated recursively, based on the results of previous iterations.

From numerical methods, we consider the method of cyclic descent of coordinates to simplify the work. However, this method only defines the local minimum, so it is important to set a good initial point. According to this method multi-dimensional optimization is reduced to some number of one-dimensional optimization. With each iteration, the point gets closer to the point of the local minimum.

For one-dimensional optimization, we can use the appropriate algorithms. Our function is not monotonous, so the usual ways to find its minimum will not work. Therefore, we will solve this problem by iterating, calculating the target function for each parameter value. Among them we will select the smallest value and the one dimensional optimization will be completed.
Optimization is finished when the vector of optimized parameters stops changing. To do this, at the end of the cycle, we need to compare this vector with what was before the last cycle. If the vectors are equal, the optimization is complete. If they differ, a new cycle begins.

Each time we start considering a new parameter value, we need to calculate the load. To do this, we need to cycle through all points on the map and find data rate in each point from each access point.

We found the data rate at every point on the map. Now we place subscribers in places where the data rate is minimal, calculate subscriber loads and find loads on access points. Select the access point with the maximum load, this load will be the value of the target function.

\section{FUNCTIONAL TEST}

The test will be performed on the example of the WiFi network of the RTS Department of KNRTU-KAI. This network includes 2 access points, works with $802.11 \mathrm{n}$ standard at a frequency of $2.4 \mathrm{GHz}$ with a band of 20 $\mathrm{MHz}$.

There are 64 subcarriers in the $20 \mathrm{MHz}$ band for the $802.11 \mathrm{n}$ standard. Some of the subcarriers form a protective interval, leaving 57 subcarriers. Of these, subcarriers numbered $7,17,28,39$, and 49 are used as pilots. They do not carry information, so their contribution to the overall data rate will be considered equal to zero.

The technical rate is determined from the duration of the OFDM symbol. For our case, this duration is $4 \mu \mathrm{s}$, so $V^{\text {tech }}=250000$. We consider the maximum possible error probability to be equal $P_{\max }^{E r}=10^{-6}$.

There is a 3D plan of the territory where the placement points are marked and all the walls with their planes are indicated. Each face is defined as a set of segments, and a normal to the plane of the face is also set. The plan includes 1153 points and 102 walls and obstacles. The network territory is fixed and does not change. The grid pitch was 1 meter.

Access points are located above the location of users by 1.5 meters.

The number of subscriber devices is 20, and the specified data rate is $2.1 \mathrm{Mbit} / \mathrm{s}$. Used access points WEP-12ac have a maximum transmitter power of 19 $\mathrm{dBm}$, receiver sensitivity of $-98 \mathrm{dBm}$ at $2.4 \mathrm{GHz}$ and -94 $\mathrm{dBm}$ at $5 \mathrm{GHz}$ [13]. The transmitter power will vary between 14 and $19 \mathrm{dBm}$ in $1 \mathrm{dBm}$ increments.

Assume that the radiation pattern of access point antennas and subscriber devices are Omnidirectional. Then the signal strength is defined as the difference between the transmitter power and losses in the propagation of radio waves.

For each access point, we selected 3 optimized parameters: $\mathrm{X}$ coordinate, $\mathrm{Y}$ coordinate and transmitter 
power. Since we use 2 access points, we get 6 parameters. Then the vector of parameters:

$$
\vec{V}=\left(x_{1}, y_{1}, x_{2}, y_{2}, P_{1}, P_{2}\right) .
$$

Since optimization is performed as a set of onedimensional optimizations, the order of parameters matters. First, we optimize the coordinates, then the power of the transmitter. Coordinate optimization is performed sequentially first for one access point, then for another. The order of parameters for optimization as in (12).

Based on the initial placement plan, access points are located by coordinates $(8,28)$ and $(8,72)$. The height of the suspension relative to the client devices is 1.5 meters.

According to recommendation ITU-R P.1238-8 [11] the following parameters were taken: $N-28-$ power loss coefficient at a distance, $L_{f}(n)=\frac{7}{2} \cdot(n-1)$ since most of the walls are wooden and are double according to the plan of the building. The distance $d_{l m}$ is determined by the Pythagorean theorem.

To determine the intersection with the wall, consider the intersection with the faces of the wall. For the segment connecting the subscriber and the access point, we make up the equation of the straight line, for the wall face, we make up the equation of the plane. We find the crossing point of a straight line and a plane, then determine whether it belongs to the segment and face. If there was an intersection with a wall face, we assume that the intersection with the wall occurred. In this model, only the fact of crossing with the wall is used, the path traversed in the wall is not taken into account.

The algorithm was implemented as a program written using the Python and $\mathrm{C}$ languages.

\section{RESULtS}

Our task was to optimize the network on the territory of the Department. To perform optimization in the way described above, we must set initial approximations. This was done as part of the preliminary phase of frequency planning.

Initially consider a network at a frequency of 2.4 $\mathrm{GHz}$. Before optimization, access points were located at coordinates $(8,28)$ and $(8,72)$. The load on access points was 2.83 and 0.11 , and the potential throughput was $299.6 \mathrm{Mbit} / \mathrm{s}$

After optimization, access points were located at coordinates $(9,28)$ and $(7,55)$. The load on access points was 0.94 and 0.91 , throughput was $459.3 \mathrm{Mbit} / \mathrm{s}$.

Comparison between subscriber data rates before and after optimization for network at a frequency of $2.4 \mathrm{GHz}$ is shown at Figure 1.

If switched network to a frequency of $5 \mathrm{GHz}$, the method continues to work, but provides lower data rate.

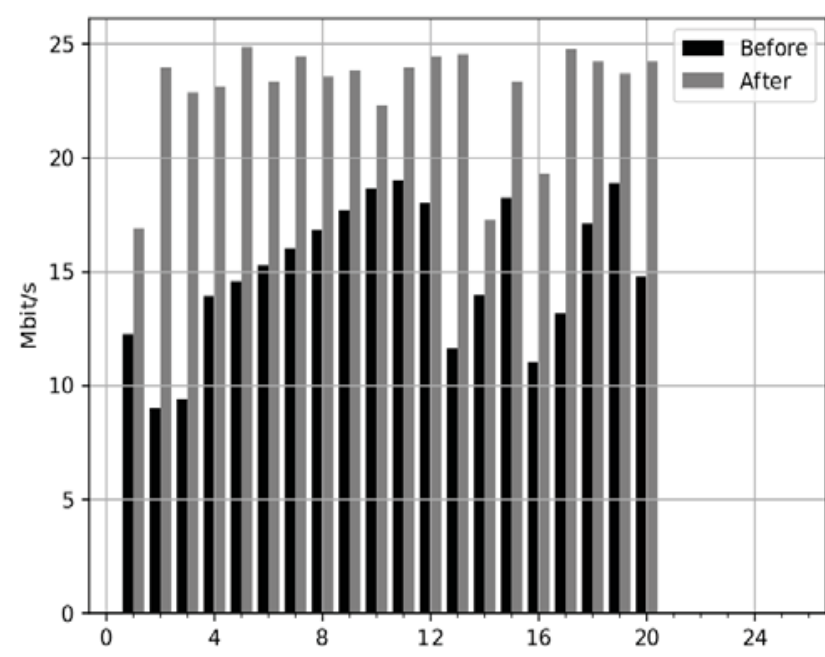

Fig. 1. The data rate distribution of users $(2.4 \mathrm{GHz})$

Before optimization at $5 \mathrm{GHz}$, access points were also located at coordinates $(8,28)$ and $(8,72)$. The load on access points was 8.54 and 0.00 , throughput was 104 Mbit/s.

After optimization at $5 \mathrm{GHz}$, access points were also located at coordinates $(9,28)$ and $(7,55)$. The load on access points was 2.53 and 2.45 , throughput was 171.3 Mbit/s.

Comparison between subscriber data rates before and after optimization for network at a frequency of $5 \mathrm{GHz}$ is shown at Figure 2.

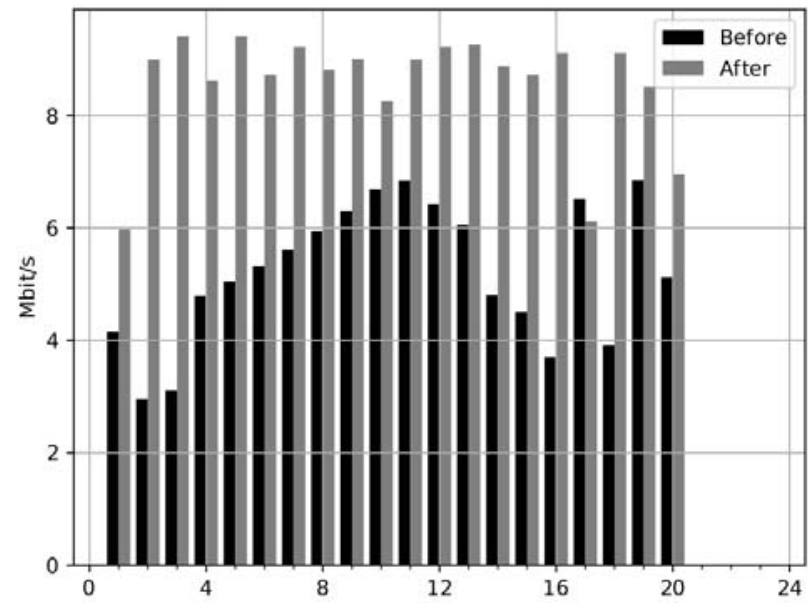

Fig. 2. The data rate distribution of users $(5 \mathrm{GHz})$

In both cases, the coordinate descent occurred in 2 cycles. The optimization time was 3 minutes.

The lower network data rates at a frequency of $5 \mathrm{GHz}$ are explained by the dependence of the losses during radio wave propagation on the frequency. 


\section{CONCLUSION}

The developed network optimization algorithm and the software that implements it were created. Network parameters optimization was performed at a frequency of $2.4 \mathrm{GHz}$, and the use of this algorithm for our network allowed us to reduce the load from 2.83 to 0.94 and increase the network throughput from 299.6 to 459.3 Mbit/s. At $5 \mathrm{GHz}$, two access points are not enough to cover the required area, but the method continues to work.

\section{REFERENCES}

1. Vybornov O.V., Izmailov A.M., Kozlov S.V.,Spirina E.A. "Electromagnetic compatibility testing for the Infinet standard 802.11n equipment", Vestnik KGTU im. A.N.Tupoleva, 2012, Vol 68. No 4-2, Pp. 160-163. (In Russian)

2. Ekahau Pro https://www.ekahau.com/products/ekahau-sitesurvey/overview/

3. Spirina E.A., Kozlov S.V., "Application efficiency analysis of the integrated optimization method frequency-territorial planning procedure on the Wi-Fi network segment", 2019 Systems of Signal Synchronization, Generating and Processing in Telecommunications, SYNCHROINFO 2019, DOI: 10.1109/SYNCHROINFO.2019.8814204

4. Spirina E.A. Frequency-territorial planning procedure for integrated optimization method. Vestnik KGTU im. A.N.Tupoleva, 2018, No 2, Pp. 115-120. (In Russian)

5. Vybornov O.V., Izmailov A.M., Kozlov S.V., Lavrushev V.N., Spirina E.A. "Prognosticating potential loading of sectors of broadband wireless access networks on the analysis of the signal to noise ratio by using GIS technology", Vestnik KGTU im. A.N.Tupoleva, 2013, Vol 69. No 4, Pp. 130-135. (In Russian)
6. Petrova E.A. "Assessment of the guaranteed rate of information transfer in the broadband radio networks taking into account intrasystem interference", Zhurnal radiojelektroniki. 2014. No 10. Available at http://jre.cplire.ru/jre/oct14/7/text.pdf. (in Russian)

7. Vintenkova Y.S., Kozlov S.V., Spirina E.A., "The estimation of data transfer rates in the broadband radio access networks with collective dynamic routing", 2017 Systems of Signal Synchronization, Generating and Processing in Telecommunications, SINKHROINFO 2017, DOI:10.1109/SINKHROINFO.2017.7997510

8. Kozlov S.V., Spirina E.A., "Application efficiency analysis of the optimal measurement algorithm for method OFDM communication networks integrated optimization", 2019 Systems of Signal Synchronization, Generating and Processing in Telecommunications, SYNCHROINFO 2019, DOI: $10.1109 /$ SYNCHROINFO.2019.8813911

9. E. A. Spirina, S. V. Kozlov. The analysis of OFDM signals optimal reception algorithms efficiency in IP networks with collective dynamic routing. Zhurnal Radioelektroniki Journal of Radio Electronics, 2017, No. 2. Available at http://jre.cplire.ru/jre/feb17/3/text.pdf. (In Russian)

10. Kozlov S.V., Spirina E.A., Fazylova L.I., "Svidetel'stvo o gosudarstvennoj registracii programmy dlja JeVM №2016663494. Programma OFDM Receiver" - Zajavka №2016661064; Registered: 18.12.2016. (In Russian)

11. Recommendation ITU-R P.1238-8 Propagation data and prediction methods for the planning of indoor radiocommunication systems and radio local area networks in the frequency range $300 \mathrm{MHz}$ to $100 \mathrm{GHz}$ https://www.itu.int/dms pubrec/itu-r/rec/p/R-REC-P.12388-201507-S!!PDF-E.pdf

12. Raislin V.I. Numerical optimization methods. Tomsk: Publishing house of the national research Tomsk Polytechnic University, 2013.

13. User manual WEP-12ac. Available at https://eltexco.ru/upload/iblock/292/WEP-

12ac_User_manual_1.19.3.pdf. 